\title{
Supernova progenitors as a test for stellar physics
}

\author{
Cyril Georgy ${ }^{1,2}$ \\ ${ }^{1}$ Geneva Observatory, Geneva University \\ Chemin des Maillettes 51, 1290 Versoix, Switzerland \\ email: cyril.georgy@unige.ch \\ ${ }^{2}$ Astrophysics Group, Keele University \\ Keele, ST5 5BG, United Kingdom
}

\begin{abstract}
The evolution of massive stars after the main sequence is not very well known from a theoretical point of view. Recent studies have shown that the way internal mixing processes, such as rotation and convection, are implemented in stellar evolution codes lead to significant discrepancies between the predictions of these codes. Particularly, the end-points of stellar evolution can be considerably different, making hard to understand the evolutionary path the lead to the observed supernovae progenitors.
\end{abstract}

Keywords. Stars: mass-loss, Stars: supergiants, Stars: luminosity function

Recent studies (e.g. Martins \& Palacios 2013) have shown that the evolution of massive stars, particularly during the advanced stages, is poorly known from a theoretical point of view. We have recently shown (Saio et al. 2013) that the pulsational properties of $\alpha$ Cyg variables provide a good test for stellar evolution models: to reproduce the observed pulsation periods, the models have to cross the Hertszprung-Russell diagram a second time after the red supergiant phase, helped by the very strong mass-loss rates during this phase. However, these models are not able to reproduce the observed surface abundances for these stars.

In Georgy et al. (2014), we have shown that using the Ledoux criterion for convection instead of the Schwartzschild one allows to improve the match between the observed and predicted surface abundances. Indeed, these criteria do not locate the convective region associated to the hydrogen shell-burning at the same depth inside the star, modifying the way chemical elements are transported towards the stellar surface. $\alpha$ Cyg variables are thus valuable targets to help constraining and understanding stellar physics.

\section{Acknowledgements}

CG acknowledges support from the European Research Council under the European Union's Seventh Framework Programme (FP/2007-2013) / ERC Grant Agreement n. 306901.

\section{References}

Georgy, C., Saio, H., \& Meynet, G. 2014, MNRAS, 439, L6

Martins, F. \& Palacios, A. 2013, A\& A, 560, A16

Saio, H., Georgy, C., \& Meynet, G. 2013, MNRAS, 433, 1246 